\title{
A phase I/II study of poziotinib combined with paclitaxel and trastuzumab in patients with HER2-positive advanced gastric cancer
}

\author{
Tae-Yong Kim ${ }^{1}$ - Hye Sook Han ${ }^{2} \cdot$ Keun-Wook Lee ${ }^{3}$. Dae Young Zang ${ }^{4}$ Sun Young Rha ${ }^{5}$. Young lee Park ${ }^{6}$. \\ Jin-Soo Kim ${ }^{7}$ Kyung-Hun Lee ${ }^{1}$. Se Hoon Park ${ }^{8}$ Eun-Kee Song ${ }^{9}$. Soo-A Jung ${ }^{10}$. NaMi Lee $^{10}$. Yeul Hong Kim ${ }^{11}$. \\ Jae Yong $\mathrm{Cho}^{12} \cdot$ Yung-Jue Bang ${ }^{1}$
}

Received: 18 December 2018 / Accepted: 27 March 2019 / Published online: 3 April 2019

(c) The International Gastric Cancer Association and The Japanese Gastric Cancer Association 2019

\begin{abstract}
Background Poziotinib (HM781-36B) is an irreversible pan-HER tyrosine kinase inhibitor which targets EGFR, HER2, and HER4. This prospective, multicenter, open-label, phase I/II study determined the maximum tolerated dose (MTD) and evaluated the safety and efficacy of poziotinib combined with paclitaxel and trastuzumab in patients with HER2-positive advanced gastric cancer (GC).

Methods Patients with HER2-positive GC previously treated with one line of chemotherapy received oral poziotinib (8 $\mathrm{mg}$ or $12 \mathrm{mg}$ ) once daily for 14 days, followed by 7 days off. Paclitaxel $\left(175 \mathrm{mg} / \mathrm{m}^{2}\right.$ infusion) and trastuzumab (8 $\mathrm{mg} / \mathrm{kg}$ loading dose, then $6 \mathrm{mg} / \mathrm{kg}$ infusion) were administered concomitantly with poziotinib on day 1 every 3 weeks.

Results In the phase I part, 12 patients were enrolled (7 at dose level 1, 5 at dose level 2). One patient receiving poziotinib $8 \mathrm{mg}$ and 2 receiving poziotinib $12 \mathrm{mg}$ had dose-limiting toxicities (DLTs); all DLTs were grade 4 neutropenia, one with fever. The most common poziotinib-related adverse events were diarrhea, rash, stomatitis, pruritus and loss of appetite. The MTD of poziotinib was determined to be $8 \mathrm{mg} /$ day and this was used in the phase II part which enrolled 32 patients. Two patients (6.3\%) had complete responses and 5 (15.6\%) had partial responses (objective response rate 21.9\%). Median progressionfree survival and overall survival were 13.0 weeks (95\% CI 9.8-21.9) and 29.5 weeks (95\% CI 17.9-59.2), respectively.

Conclusions The MTD of poziotinib combined with paclitaxel and trastuzumab was $8 \mathrm{mg} /$ day. This combination yielded promising anti-tumor efficacy with manageable toxicity in previously treated patients with HER2-positive GC.
\end{abstract}

Keywords Chemotherapy $\cdot$ Gastric cancer $\cdot$ HER2 $\cdot$ Poziotinib $\cdot$ Trastuzumab

Electronic supplementary material The online version of this article (https://doi.org/10.1007/s10120-019-00958-4) contains supplementary material, which is available to authorized users.

Yung-Jue Bang

bangyj@snu.ac.kr

1 Department of Internal Medicine, Seoul National University Hospital, Seoul National University College of Medicine, 101 Daehak-ro, Jongno-gu, Seoul 03080, South Korea

2 Chungbuk National University College of Medicine, Cheongju, South Korea

3 Seoul National University Bundang Hospital, Seoul National University College of Medicine, Seongnam, South Korea

4 Hallym University Sacred Heart Hospital, Anyang-si, South Korea

5 Yonsei Cancer Center, Yonsei University College of Medicine, Seoul, South Korea
6 National Cancer Center, Seoul, South Korea

7 Seoul National University Boramae Medical Center, Seoul, South Korea

8 Sungkyunkwan University Samsung Medical Center, Seoul, South Korea

9 Chonbuk National University Medical School, Jeonju, South Korea

10 Hanmi Pharmaceutical Co., Ltd., Seoul, South Korea

11 Korea University Anam Hospital, Seoul, South Korea

12 Gangnam Severance Hospital, Seoul, South Korea 


\section{Introduction}

Gastric cancer (GC) is the fifth most common cancer and the third leading cause of cancer-related death worldwide [1]. Chemotherapy is the backbone of treatment for most patients with metastatic or recurrent GC. While there is no universally accepted chemotherapy regimen for these patients, fluoropyrimidine and platinum doublet is used most commonly worldwide. Some physicians add docetaxel or epirubicin on top of doublet; however, triplets are not as popular, due to toxicity concerns [2].

Human epidermal growth factor receptor 2 (HER2) is part of a family of receptors associated with tumor cell proliferation, apoptosis, adhesion, migration, and differentiation [3]. HER 2 is reported to be amplified in 13-22\% of GC $[4,5]$. It has been shown that patients with HER2positive GC benefited substantially from trastuzumab add-on chemotherapy, and trastuzumab combined with doublet of fluoropyrimidine and platinum has become the standard of care in patients with metastatic or unresectable HER2-positive GC [6]. However, most patients eventually develop resistance to trastuzumab, and new therapeutic approaches are necessary.

HER2 activation resulted in increased epidermal growth factor receptor (EGFR) signaling in a preclinical study, suggesting that dual inhibition of HER 2 and EGFR could overcome resistance to trastuzumab [7]. Lapatinib is a tyrosine kinase inhibitor (TKI) that targets the tyrosine kinases of both HER2 and EGFR, and the combination of lapatinib and capecitabine was effective in HER2-positive breast cancer [8]. Lapatinib in combination with capecitabine and oxaliplatin as first line, or with paclitaxel as second line, was evaluated in patients with HER2-positive GC $[9,10]$. However, these two trials failed to meet the primary endpoint. HER subfamily members should synergistically interact with the same or another HER subfamily member, suggesting that pan-HER inhibitors could be more potent than those which inhibit only one or two HER subfamily receptors [11-13].

Poziotinib (HM781-36B) is an irreversible pan-HER TKI, which targets EGFR, HER2, and HER4. Poziotinib showed strong in vitro and in vivo anti-tumor activity in HER2-amplified GC cells and also demonstrated a synergistic inhibitory effect when combined with chemotherapeutic agents, including paclitaxel [14]. In a phase I study of poziotinib monotherapy, the maximum tolerated dose (MTD) was $18 \mathrm{mg} /$ day, with continuous dosing, and $24 \mathrm{mg} /$ day with intermittent dosing (14 days on, 7 days off) [15]. The main dose-limiting toxicities (DLTs) were grade 3 diarrhea with intermittent and continuous dosing, and one patient had grade 3 anorexia with continuous dosing. Commonly observed treatment-emergent adverse events (TEAEs) were diarrhea, rash, stomatitis, pruritus, and anorexia. In the aforementioned phase I study, poziotinib monotherapy produced response rates of $16 \%$ and $21 \%$ with intermittent and continuous dosing, respectively, in patients with advanced solid tumors including HER2amplified GC and breast cancer.

The combination of trastuzumab plus lapatinib enhanced the response to a taxane, and the triplet regimen with trastuzumab, lapatinib and a taxane showed marked inhibitory potency in HER2-positive breast cancer cells [16]; this approach was also effective in patients with HER2-positive breast cancer [17]. Based on the aforementioned preclinical data, the present trial was designed to explore the clinical efficacy of the pan-HER inhibitor, poziotinib, combined with trastuzumab and paclitaxel in patients with HER2-positive GC as second-line treatment.

The objectives of the current phase I/II study were to determine the MTD and the recommended phase II dose (RP2D) of poziotinib, when combined with paclitaxel and trastuzumab (phase I part), and to assess the anti-tumor efficacy of this combination in previously treated patients with HER2-positive advanced GC (phase II part).

\section{Patients and methods}

This was a prospective phase I/II study, conducted in 11 centers in Korea.

\section{Patients}

Key eligibility criteria included: patients aged $\geq 19$ years; histopathologically confirmed locally advanced unresectable, relapsed or metastatic gastric adenocarcinoma (including adenocarcinoma of the esophagogastric junction); HER2 immunohistochemistry (IHC) $3+$ or HER 2 IHC $2+$ and HER2 fluorescence in situ hybridization (FISH) +; the presence of one or more measurable lesions by Response Evaluation Criteria in Solid Tumors (RECIST, version 1.1) [18]; one previous line of chemotherapy, including fluoropyrimidine or platinum, regardless of trastuzumab exposure; and adequate bone marrow and hepatic functions. Key exclusion criteria included: a history of hypersensitivity to drugs containing Cremophor ${ }^{\circledR}$ EL and trastuzumab; previous exposure to taxanes, and symptomatic central nervous system metastasis.

This study was conducted in accordance with the Declaration of Helsinki and the International Conference on Harmonization Good Clinical Practice Guidelines and was approved by the Institutional Review Board of each participating center. All patients provided written informed consent before enrollment. 


\section{Treatment procedures}

Each 21-day cycle consisted of orally administered poziotinib once daily for 14 days, in combination with paclitaxel $\left(175 \mathrm{mg} / \mathrm{m}^{2}\right.$ infusion) and trastuzumab $(8 \mathrm{mg} / \mathrm{kg}$ loading dose, followed by $6 \mathrm{mg} / \mathrm{kg}$ infusion) on day 1 . In the phase I part of the study, increasing doses of poziotinib $(8 \mathrm{mg}$, $12 \mathrm{mg}$ or $16 \mathrm{mg}$ ) were tested to determine the RP2D of poziotinib, in combination with paclitaxel and trastuzumab. DLTs were evaluated in 6 patients at each poziotinib dose level. If DLT was observed in $\leq 1$ patient, dose escalation was continued to the next dose level. If DLT was observed in $\geq 2$ patients, dose escalation was stopped and the MTD was determined as the highest level at which DLT occurred in $\leq 1$ patient. In the phase II part of the study, 32 patients were treated with the RP2D of poziotinib, in combination with paclitaxel and trastuzumab.

\section{Safety analyses and efficacy}

Adverse events (AEs) and TEAEs were evaluated using National Cancer Institute-Common Terminology Criteria for Adverse Events (version 4.03). DLTs were defined as grade $\geq 3$ non-hematologic toxicities (except for alopecia); grade $\geq 3$ diarrhea, nausea and vomiting, despite maximal dosage of anti-diarrhea and/or anti-emetic medication (as applicable); and grade 4 neutropenia sustained for $\geq 7$ days, grade 3-4 neutropenia with fever or infection, grade 4 thrombocytopenia and grade 3 thrombocytopenia sustained for $\geq 7$ days, or with accompanying bleeding, or requiring transfusion. Left ventricular ejection fraction (LVEF) assessment was performed at baseline, on day 1 of the first 3 cycles and every 3 cycles thereafter. All patients who received at least one dose of poziotinib were included in the safety analysis. Tumor response was evaluated by CT or MRI of the thorax, abdomen and pelvis every 6 weeks using RECIST version 1.1 , according to investigator assessment.

\section{Statistical analysis}

The primary objective of phase I was to assess the safety and tolerability of poziotinib and to determine the MTD of poziotinib when combined with paclitaxel and trastuzumab. The primary objective of the phase II part was to assess the objective response rate (ORR) of poziotinib combined with paclitaxel and trastuzumab. The secondary objectives were to evaluate the safety and tolerability, progression-free survival (PFS), time to tumor progression (TTP) and duration of overall response (DOR).

For phase II, we hypothesized that an ORR $<5 \%$ would be ineffective and an ORR $>20 \%$ would be considered to be clinically significant. Sample size was calculated using Simon's 2-stage minimax design [19], with $80 \%$ power and a significance level of 5\%. Of the total 27 target subjects, 13 were required in the 1 st stage. If the tumor response was 0 out of 13 patients, the study was planned to be terminated early. However, if at least 1 response was observed, an additional 14 patients would be enrolled in the study.

\section{Results}

\section{Patients' characteristics}

From January 2013 to August 2014, a total of 44 patients (12 in phase I and 32 in phase II) were enrolled from 11 sites in Korea. The baseline characteristics of the study population are summarized in Table 1. Median age was 61.5 (range $35.0-76.0)$, and $38(86.4 \%)$ were males. Main metastatic sites were lymph node (70.5\%) and liver (63.6\%). Out of 44 patients, $33(75.0 \%)$ were previously treated with a trastuzumab-containing regimen.

\section{Phase I part}

\section{Determination of the MTD}

Seven patients were enrolled at dose level 1 (poziotinib $8 \mathrm{mg}$ ); however, one patient was not evaluable for DLT, due to loss of follow-up before completion of the first cycle. Among 6 patients, DLT was observed in 1 (grade 4 neutropenia). At dose level 2 (poziotinib $12 \mathrm{mg}$ ), 5 patients were enrolled, and DLT was observed in 2 (one with grade 4 neutropenia and the other with febrile neutropenia with grade 4 neutropenia) (Table 2). Therefore, poziotinib $8 \mathrm{mg}$ was determined to be the MTD.

\section{Toxicity and dose adjustments}

The safety analysis set consisted of 12 patients in phase I. Grade 3 or higher neutropenia and febrile neutropenia were observed in $9(75.0 \%)$ and $2(16.7 \%)$ patients, respectively (Online Resource 1). The most common poziotinib-related AEs were diarrhea, rash, stomatitis, pruritus, and loss of appetite (Table 3 ). Four (33.3\%) experienced Grade 3 or higher diarrhea. One had a $\geq 15 \%$ decline in LVEF from baseline with an absolute value $<50 \%$ at dose level 2 . Poziotinib dose reduction from 8 to $6 \mathrm{mg}$ was performed in 1 (14.3\%) of 7 patients at dose level 1 and reduction from 12 to $8 \mathrm{mg}$ was observed in $4(80.0 \%)$ of 5 patients at dose level 2. Dose reduction and discontinuation of paclitaxel were observed in $2(28.6 \%)$ and $1(14.3 \%)$ patients at dose level 1 and $1(20.0 \%)$ and $4(80.0 \%)$ at dose level 2, respectively (Online Resource 2). 
Table 1 Summary of baseline characteristics of the study population

\begin{tabular}{|c|c|c|c|c|c|}
\hline & \multicolumn{3}{|l|}{ Phase I } & \multirow{2}{*}{$\begin{array}{l}\text { Phase II } \\
\text { RD: } 8 \mathrm{mg}(n=32)\end{array}$} & \multirow{2}{*}{$\begin{array}{l}\text { Total population } \\
\text { Total }(n=44)\end{array}$} \\
\hline & $8 \mathrm{mg}(n=7)$ & $12 \mathrm{mg}(n=5)$ & Total $(n=12)$ & & \\
\hline \multicolumn{6}{|l|}{ Age (years) } \\
\hline Median (range) & $61.0(41.0,74.0)$ & $57.0(45.0,73.0)$ & $59.0(41.0,74.0)$ & $62.5(35.0,76.0)$ & $61.5(35.0,76.0)$ \\
\hline \multicolumn{6}{|l|}{ Sex, $n(\%)$} \\
\hline Male & $6(85.7)$ & $5(100.0)$ & $11(91.7)$ & $27(84.4)$ & $38(86.4)$ \\
\hline Female & $1(14.3)$ & 0 & $1(8.3)$ & $5(15.6)$ & $6(13.6)$ \\
\hline \multicolumn{6}{|l|}{ ECOG PS, $n(\%)$} \\
\hline 0 & $6(85.7)$ & $1(20.0)$ & $7(58.3)$ & $12(37.5)$ & $19(43.2)$ \\
\hline 1 & $1(14.3)$ & $4(80.0)$ & $5(41.7)$ & $19(59.4)$ & $24(54.5)$ \\
\hline 2 & 0 & 0 & 0 & $1(3.1)$ & $1(2.3)$ \\
\hline \multicolumn{6}{|c|}{ Metastasis at initial diagnosis, $n(\%)$} \\
\hline Yes & $6(85.7)$ & $3(60.0)$ & $9(75.0)$ & $22(68.7)$ & $31(70.5)$ \\
\hline No & $1(14.3)$ & $2(40.0)$ & $3(25.0)$ & $10(31.3)$ & $13(29.5)$ \\
\hline \multicolumn{6}{|c|}{ Primary tumor location at diagnosis, $n(\%)$} \\
\hline Stomach & $7(100.0)$ & $5(100.0)$ & $12(100.0)$ & $30(93.7)$ & $42(95.4)$ \\
\hline Gastroesophageal junction & 0 & 0 & 0 & $2(6.3)$ & $2(4.6)$ \\
\hline \multicolumn{6}{|c|}{ Location of the metastatic lesion at the screening of study } \\
\hline Lung & $2(28.6)$ & $1(20.0)$ & $3(25.0)$ & $9(28.1)$ & $12(27.3)$ \\
\hline Lymph node & $6(85.7)$ & $4(80.0)$ & $10(83.3)$ & $21(65.6)$ & $31(70.5)$ \\
\hline Liver & $5(71.4)$ & $3(60.0)$ & $8(66.7)$ & $20(62.5)$ & $28(63.6)$ \\
\hline Peritoneum & $1(14.3)$ & $1(20.0)$ & $2(16.7)$ & $13(40.6)$ & $15(34.1)$ \\
\hline Bone & $1(14.3)$ & $1(20.0)$ & $2(16.7)$ & $2(6.3)$ & $4(9.1)$ \\
\hline Brain & 0 & 0 & 0 & $1(3.1)$ & $1(2.3)$ \\
\hline \multicolumn{6}{|l|}{ Histologic type at diagnosis } \\
\hline Adenocarcinoma & $6(85.7)$ & $3(60.0)$ & $9(75.0)$ & $27(84.4)$ & $36(81.8)$ \\
\hline SRCC & $1(14.3)$ & 0 & $1(8.3)$ & $3(9.4)$ & $4(9.1)$ \\
\hline Mixed type & 0 & $2(40.0)$ & $2(16.7)$ & $1(3.1)$ & $3(6.8)$ \\
\hline Unknown & 0 & 0 & 0 & $1(3.1)$ & $1(2.3)$ \\
\hline \multicolumn{6}{|l|}{ HER2 status } \\
\hline IHC $3+$ & $6(85.7)$ & $3(60.0)$ & $9(75.0)$ & $21(65.6)$ & $30(68.2)$ \\
\hline IHC $2+$ and FISH+ & $1(14.3)$ & $2(40.0)$ & $3(25.0)$ & $11(34.4)$ & $14(31.8)$ \\
\hline \multicolumn{6}{|l|}{ Previous gastrectomy } \\
\hline Yes & $3(42.9)$ & $1(20.0)$ & $4(33.3)$ & $9(28.1)$ & $13(29.5)$ \\
\hline No & $4(57.1)$ & $4(80.0)$ & $8(66.7)$ & $23(71.9)$ & $31(70.5)$ \\
\hline \multicolumn{6}{|l|}{ Adjuvant chemotherapy } \\
\hline Yes & $1(14.3)$ & $1(20.0)$ & $2(16.7)$ & 7 (21.9) & $9(20.5)$ \\
\hline No & $6(85.7)$ & $4(80.0)$ & $10(83.3)$ & $25(78.1)$ & $35(79.5)$ \\
\hline \multicolumn{6}{|c|}{ Previous exposure of trastuzumab } \\
\hline Yes & $4(57.1)$ & $5(100.0)$ & $9(75.0)$ & $24(75.0)$ & $33(75.0)$ \\
\hline No & $3(42.9)$ & 0 & $3(25.0)$ & $8(25.0)$ & $11(25.0)$ \\
\hline
\end{tabular}

ECOG Eastern Cooperative Oncology Group, PS performance status, IHC immunohistochemistry, FISH fluorescence in situ hybridization, $S R C C$ signet ring cell carcinoma

\section{Efficacy}

Four patients $(33.3 \%$; 95\% CI 9.9-65.1) had partial responses (Table 4). The disease control rate (DCR; partial response + stable disease) was $66.7 \%$ (95\% CI 34.9-90.1). Median PFS and OS were 17.7 weeks (95\% CI 5.4-30.2) and 30.6 weeks (95\% CI 12.2-195.0), respectively (Online Resource 3 and 4). 
Table 2 Summary of doselimiting toxicities

\begin{tabular}{llll}
\hline Safety set & \multicolumn{2}{l}{ Phase I } & \\
\cline { 2 - 4 } & \multicolumn{1}{c}{$12 \mathrm{mg}(n=5)$} & Total $(n=12)$ \\
\hline Dose-limiting toxicities (DLTs), $n(\%)$ & & $3(25.0)$ \\
DLTs & $1(14.3)$ & $2(40.0)$ & \\
& Grade 4 neutropenia & Grade 4 neutropenia for $\geq 7$ days & \\
& for $\geq 7$ days & Grade 4 neutropenia with fever &
\end{tabular}

${ }^{\text {a Out of }} 7$ subjects, 6 were available for assessment DLTs because 1 had loss of follow-up before completion of the first cycle

\begin{tabular}{|c|c|c|c|c|}
\hline \multirow[t]{2}{*}{ System organ class preferred term } & \multicolumn{3}{|l|}{ Phase I } & \multirow{2}{*}{$\begin{array}{l}\text { Phase II } \\
\text { RD: } 8 \mathrm{mg}(n=32)\end{array}$} \\
\hline & $8 \mathrm{mg}(\mathrm{n}=7)$ & $12 \mathrm{mg}(n=5)$ & Total $(n=12)$ & \\
\hline \multicolumn{5}{|l|}{ Gastrointestinal disorder } \\
\hline Diarrhea & $7(100.0)$ & $5(100.0)$ & $12(100.0)$ & $29(90.6)$ \\
\hline Stomatitis & $4(57.1)$ & $2(40.0)$ & $6(50.0)$ & $13(40.6)$ \\
\hline Nausea & $1(14.3)$ & $1(20.0)$ & $2(16.7)$ & $3(9.4)$ \\
\hline Vomiting & 0 & 0 & 0 & $4(12.5)$ \\
\hline \multicolumn{5}{|l|}{ Skin and subcutaneous tissue disorders } \\
\hline Rash & $4(57.1)$ & $3(60.0)$ & $7(58.3)$ & $22(68.8)$ \\
\hline Pruritus & $1(14.3)$ & $1(20.0)$ & $2(16.7)$ & $7(21.9)$ \\
\hline Dermatitis acneiform & $2(28.6)$ & 0 & $2(16.7)$ & 0 \\
\hline \multicolumn{5}{|l|}{ Metabolism and nutrition disorders } \\
\hline Decreased appetite & 0 & $1(20.0)$ & $1(8.3)$ & $14(43.8)$ \\
\hline Hypophagia & 0 & $2(40.0)$ & $2(16.7)$ & $1(3.1)$ \\
\hline Hypokalemia & $1(14.3)$ & $1(20.0)$ & $2(16.7)$ & $1(3.1)$ \\
\hline Dehydration & $1(14.3)$ & $1(20.0)$ & $2(16.7)$ & 0 \\
\hline \multicolumn{5}{|c|}{ General disorders and administration site conditions } \\
\hline Mucosal inflammation & $1(14.3)$ & $2(40.0)$ & $3(25.0)$ & $3(9.4)$ \\
\hline Fatigue & 0 & 0 & 0 & $7(21.9)$ \\
\hline \multicolumn{5}{|c|}{ Musculoskeletal and connective tissue disorders } \\
\hline Myalgia & 0 & 0 & 0 & $4(12.5)$ \\
\hline \multicolumn{5}{|l|}{ Psychiatric disorders } \\
\hline Insomnia & $2(28.6)$ & 0 & $2(16.7)$ & 0 \\
\hline
\end{tabular}

Table 3 Safety profiles: adverse events related to poziotinib ( $\geq 10 \%$ of patients)

\section{Phase Il part}

\section{Safety analysis}

Grade 3 or higher TEAEs neutropenia and febrile neutropenia developed in $4(12.5 \%)$ and $3(9.4 \%)$ of patients, respectively (Online Resource 1). The most common poziotinib-related TEAEs were diarrhea, rash, stomatitis and decreased appetite, similar to results obtained in phase I (Table 3). Grade 3 or higher diarrhea was observed in 13 patients $(40.6 \%)$ (Online Resource 1). Four patients (12.5\%) discontinued the study before the first assessment due to treatment-related toxicity. There was no grade 3 or 4 left ventricular systolic dysfunction (LVSD). Twenty $(62.5 \%)$ patients received a reduced dose of poziotinib. A reduced dose of paclitaxel was administered to 12 patients $(37.5 \%)$ and paclitaxel was discontinued in 3 patients (9.4\%) (Online Resource 2).

\section{Efficacy}

Twenty patients (62.5\%) had tumor shrinkage (Fig. 1). Confirmed response was observed in 7 patients $(21.9 \%$; 95\% CI 9.3-40.0). Two patients (6.3\%) had complete responses and $5(15.6 \%)$ had partial responses. DCR was 71.9\% (95\% CI 53.3-86.3) (Table 4). The median PFS was 13.0 weeks (95\% CI 9.8-21.9) (Online Resources 
Table 4 Summary of tumor responses

\begin{tabular}{llllll}
\hline & Phase I & & & Phase II & \multicolumn{2}{c}{ Total population } \\
\cline { 2 - 3 } & $8 \mathrm{mg}$ & $12 \mathrm{mg}$ & Total & RD: $8 \mathrm{mg}$ & Total \\
\hline All patients, $n$ & 7 & 5 & 12 & 32 & 44 \\
Objective response rate, $n(\%)$ & $2(28.6)$ & $2(40.0)$ & $4(33.3)$ & $7(21.9)$ & $11(25.0)$ \\
Exact 95\% CI & $3.7-71.0$ & $5.3-85.3$ & $9.9-65.1$ & $9.3-40.0$ & $13.2-40.3$ \\
Disease control rate, $n(\%)$ & $5(71.4)$ & $3(60.0)$ & $8(66.7)$ & $23(71.9)$ & $31(70.5)$ \\
Exact 95\% CI & $29.0-96.3$ & $14.7-94.7$ & $34.9-90.1$ & $53.3-86.3$ & $54.8-83.2$ \\
Best overall response, $n(\%)$ & & & & & \\
CR & 0 & 0 & 0 & $2(6.3)$ & $2(4.5)$ \\
PR & $2(28.6)$ & $2(40.0)$ & $4(33.3)$ & $5(15.6)$ & $9(20.5)$ \\
SD & $3(42.9)$ & $1(20.0)$ & $4(33.3)$ & $16(50.0)$ & $20(45.5)$ \\
PD & $1(14.3)$ & $2(40.0)$ & $3(25.0)$ & $5(15.6)$ & $8(18.2)$ \\
Unknown & $1(14.3)$ & 0 & $1(8.3)$ & $4(12.5)$ & $5(11.4)$ \\
Trastuzumab-pretreated patients, $n$ & 4 & 5 & 9 & 24 & 33 \\
Objective response rate, $\mathrm{n}(\%)$ & $1(25.0)$ & $2(40.0)$ & $3(33.3)$ & $3(12.5)$ & $6(18.2)$ \\
Exact 95\% CI & $0.6-80.6$ & $5.3-85.3$ & $7.5-70.1$ & $2.7-32.4$ & $7.06-35.5$ \\
Disease control rate, $n(\%)$ & $3(75.0)$ & $3(60.0)$ & $6(66.7)$ & $18(75.0)$ & $24(72.7)$ \\
Exact 95\% CI & $19.4-99.4$ & $14.7-94.7$ & $29.9-92.5$ & $53.3-90.2$ & $54.5-86.7$ \\
Trastuzumab-naïve patients, $n$ & 3 & 0 & 3 & 8 & 11 \\
Objective response rate, $\mathrm{n}(\%)$ & $1(33.3)$ & 0 & $1(33.3)$ & $4(50.0)$ & $5(45.5)$ \\
Exact 95\% CI & $0.8-90.6$ & & $0.8-90.6$ & $15.7-84.3$ & $16.8-76.6$ \\
Disease control rate, $n(\%)$ & $2(66.7)$ & 0 & $2(66.7)$ & $5(62.5)$ & $7(63.6)$ \\
Exact 95\% CI & $9.4-99.2$ & & $9.4-99.2$ & $24.5-91.5$ & $30.8-89.1$ \\
\hline
\end{tabular}

$R D$ recommended dose, $C I$ confidence interval, $C R$ complete response, $P R$ partial response, $S D$ stable disease, $P D$ progressive disease

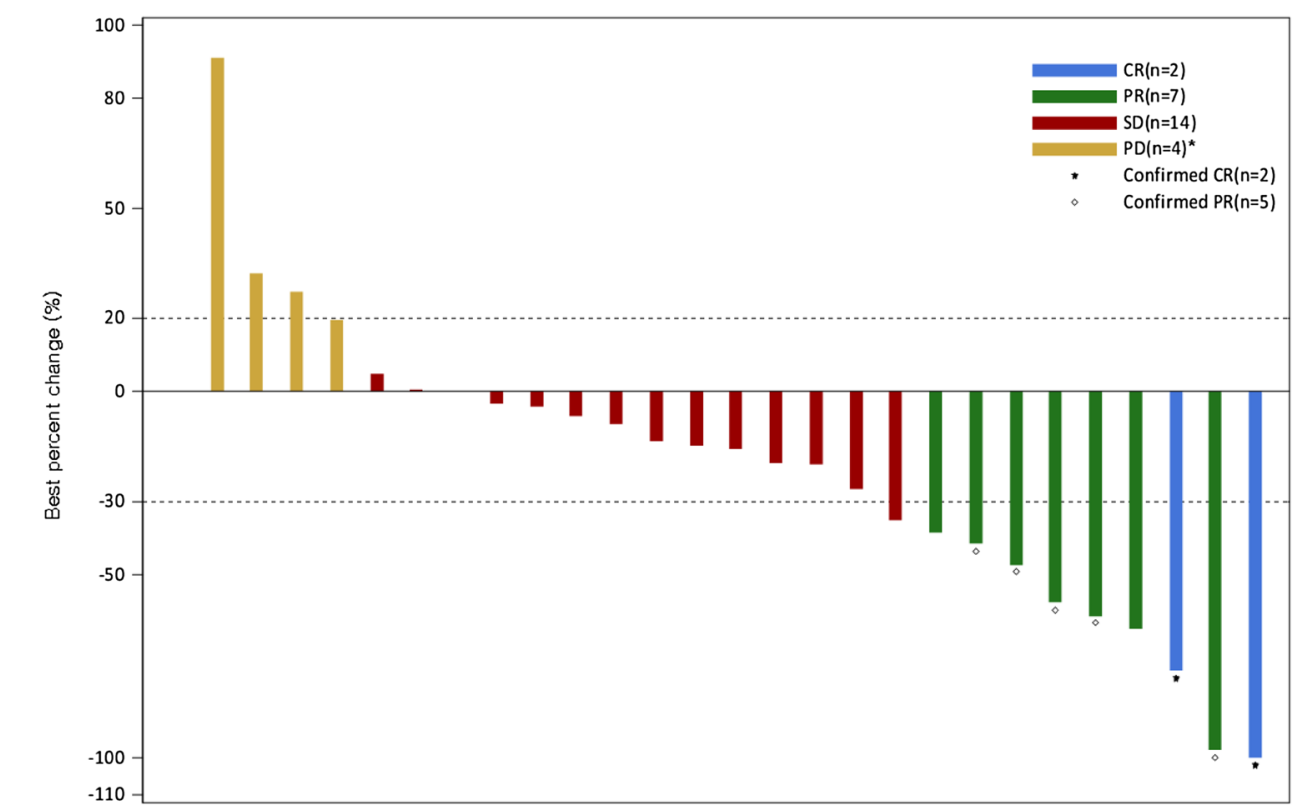

Fig. 1 Waterfall plot of best percent change in tumor diameter of target lesions in the phase II part of the study. Best percent change in target lesions $(\%)=($ the smallest post-treat ment tumor diameter - baseline tumor diameter)/baseline tumor diameter $* 100 . *$ One subject who is clinical PD is not included in this result because of no evaluation value for tumor diameter after baseline
3-5). The median OS was 29.5 weeks (95\% CI 17.9-59.2) (Online Resource 3 and 4). The median TTP and DOR were 15.0 weeks (95\% CI 10.0-23.1) and 26.8 weeks (95\% CI 17.2-71.8), respectively (Online Resource 3). When looking into clinical efficacy in terms of trastuzumab exposure, the ORRs of trastuzumab-pretreated and trastuzumab-naïve patients were $12.5 \%$ and $50.0 \%$, respectively (Table 4). The median OS and PFS were 29.5 weeks $(95 \%$ CI 17.9-40.9) and 13.0 weeks (9.8-18.7) in trastuzumabpretreated patients, and 42.6 weeks (95\% CI 3.0-111.5) 
and 18.7 weeks (95\% CI 3.0-) in trastuzumab-naïve patients (Online Resource 3).

\section{Discussion}

In this prospective, multicenter, open-label phase I/II study, poziotinib $8 \mathrm{mg}$ combined with paclitaxel plus trastuzumab showed manageable toxicity and promising efficacy in previously treated patients with advanced HER2-positive GC. To our knowledge, this study is the first to evaluate a pan-HER inhibitor combined with a chemotherapeutic agent and trastuzumab in HER2-positive tumors.

In a previous phase I study, poziotinib MTDs were determined to be $24 \mathrm{mg} /$ day with intermittent dosing (14 days on and 7 days off) and $18 \mathrm{mg} /$ day with continuous dosing [15]. In the phase I part of the current study, the MTD of poziotinib was determined to be $8 \mathrm{mg} /$ day, which is much lower than the poziotinib MTD of $24 \mathrm{mg} /$ day as monotherapy [15]. Commonly observed TEAEs in our study were diarrhea, rash, stomatitis and pruritus. All patients experienced at least one TEAE in our study, with a frequency similar to that reported in the phase I trial of poziotinib monotherapy [15]. In contrast to the phase I monotherapy trial, all DLTs in our study were reported to be grade 4 neutropenia or febrile neutropenia accompanying grade 4 neutropenia, rather than diarrhea.

In the aforementioned phase I trial, the ORR and median PFS of poziotinib monotherapy were reported to be $16 \%$ and 12.0 weeks (intermittent dosing schedule) and $21 \%$ and 9.0 weeks (continuous dosing schedule) [15]. Another pan-HER inhibitor, dacomitinib, as monotherapy produced an ORR of 7.4\% (95\% CI 0-17.5) and a median PFS of 2.1 months (95\% CI 2.3-3.4) in HER2-positive GC [20]. Given the ORR and median PFS of our study, these results suggest that pan-HER inhibitors, in combination with chemotherapy, could be more potent than pan-HER inhibitors alone.

Dual blockade of HER2 receptors has become the standard of care in HER2-positive breast cancer [21-24]. Pertuzumab in combination with docetaxel plus trastuzumab significantly increased OS in HER2-positive breast cancer [24]. However, in contrast to HER2-positive breast cancer, pertuzumab in combination with chemotherapy did not extend OS in HER2-positive GC [25]. This finding indicates that the efficacy of dual blockade in HER2-positive GC might be different from that in HER2-positive breast cancer. However, even in vitro, dual blockade with lapatinib and trastuzumab showed highly synergistic antitumor activity against HER2-amplified GC cells [26]. In the current study, poziotinib in combination with trastuzumab showed a good tumor response, suggesting that this dual blockade strategy, including pan-HER inhibitors, might be a promising treatment option in HER2-positive $\mathrm{GC}$ as salvage treatment.

The continuation of anti-HER 2 targeted therapy beyond progression is a generally accepted treatment strategy in HER2-positive breast cancer [27]. A retrospective study reported that trastuzumab beyond progression increased PFS and OS in HER2-positive GC [28]. However, in a prospective phase II study, trastuzumab beyond progression as second line failed to improve PFS and the ORR in patients with HER2-positive GC, indicating that the role of trastuzumab beyond progression remains controversial in HER2-positive GC [29].

Antibody-drug conjugate (ADC) is one of the emerging treatment strategies in HER2-positive tumors. A phase III trial of trastuzumab emtansine was performed in patients with HER2-positive GC, who failed prior fluoropyrimidine and platinum [30]. However, trastuzumab emtansine was not superior to paclitaxel. The ORR was only $20.6 \%$ (95\% CI 15.26-26.45). DS8201a, a new HER2-targeting ADC, has shown potent anti-tumor activity against HER2positive GC cells [31]. In a phase I study, DS8201a presented an ORR of $44 \%$ and DCR of $78 \%$ in patients with HER2-positive GC resistant to trastuzumab, and also in patients with low HER2-expressing GC, suggesting that HER2-targeting ADC could be a promising salvage treatment in HER2-positive GC [32, 33]).

In the current study, pretreatment biopsy was not mandatory; therefore, patients were enrolled based on HER2 expression at initial diagnosis. However, differences in HER 2 expression before and after trastuzumab were reported in HER2-positive GC and a loss of HER2 positivity was even observed in $32 \%$ of patients who received trastuzumab $[34,35]$. These observations indicate that reassessment of exact HER2 status is essential just before re-challenge with anti-HER 2 targeted therapy and, as such, this represents a limitation of our study.

In conclusion, poziotinib $(8 \mathrm{mg})$ in combination with paclitaxel and trastuzumab showed good clinical efficacy and manageable toxicity in patients with HER2-positive GC who had received one line of chemotherapy. Dual blockade inhibition using trastuzumab with a pan-HER inhibitor could be a promising strategy to overcome resistance to trastuzumab. Further clinical trials are warranted to examine the efficacy of poziotinib in combination with an HER2-targeted agent.

Acknowledgements Editorial assistance, under the guidance of the authors, was provided by David P. Figgitt PhD, ISMPP CMPPTM, Content Ed Net, with funding from Hanmi Pharmaceutical Co., Ltd., Seoul, South Korea.

Funding The study was sponsored by Hanmi. 


\section{Compliance with ethical standards}

Conflict of interest Yung-Jue Bang has served in consultant/advisory board roles for AstraZeneca, Novartis, Genentech/Roche, MSD, Bayer, BMS, Eli Lilly, Merck Serono, Taiho, Daiichi-Sankyo, Astellas, BeiGene, Green Cross, Samyang Biopharmaceuticals and Hanmi, and has received research grants from AstraZeneca, Novartis, Genentech/ Roche, MSD, Merck Serono, Bayer, GSK, BMS, Pfizer, Eli Lilly, Boeringer-Ingelheim, MacroGenics, Boston Biomedical, FivePrime, CKD Pharma, Ono, Taiho, Takeda, BeiGene, Green Cross, Curis, Daiichi Sankyo and Astellas. Soo-A Jung and NaMi Lee are employees of Hanmi Pharmaceutical Co., Ltd., Seoul, South Korea. The other authors declare no conflicts of interest.

Ethical standards All procedures followed were in accordance with the Declaration of Helsinki and the International Conference on Harmonization Good Clinical Practice Guidelines and the study was approved by the Institutional Review Board of each participating center. All patients provided written informed consent before enrollment.

\section{References}

1. Ferlay J, Soerjomataram I, Dikshit R, Eser S, Mathers C, Rebelo $\mathrm{M}$, et al. Cancer incidence and mortality worldwide: sources, methods and major patterns in GLOBOCAN 2012. Int J Cancer. 2015;136(5):E359-86.

2. Wagner AD, Syn NL, Moehler M, Grothe W, Yong WP, Tai BC, et al. Chemotherapy for advanced gastric cancer. Cochrane Database Syst Rev. 2017;8:CD004064.

3. Hudis CA. Trastuzumab-mechanism of action and use in clinical practice. N Engl J Med. 2007;357(1):39-51.

4. Gravalos C, Jimeno A. HER2 in gastric cancer: a new prognostic factor and a novel therapeutic target. Ann Oncol. 2008;19(9):1523-9.

5. Van Cutsem E, Bang YJ, Feng-Yi F, Xu JM, Lee KW, Jiao SC, et al. HER2 screening data from ToGA: targeting HER2 in gastric and gastroesophageal junction cancer. Gastric Cancer. 2015;18(3):476-84.

6. Bang YJ, Van Cutsem E, Feyereislova A, Chung HC, Shen L, Sawaki A, et al. Trastuzumab in combination with chemotherapy versus chemotherapy alone for treatment of HER2-positive advanced gastric or gastro-oesophageal junction cancer (ToGA): a phase 3, open-label, randomised controlled trial. Lancet. 2010;376(9742):687-97.

7. Henjes F, Bender C, von der Heyde S, Braun L, Mannsperger $\mathrm{HA}$, Schmidt C, et al. Strong EGFR signaling in cell line models of ERBB2-amplified breast cancer attenuates response towards ERBB2-targeting drugs. Oncogenesis. 2012;1:e16.

8. Geyer CE, Forster J, Lindquist D, Chan S, Romieu CG, Pienkowski T, et al. Lapatinib plus capecitabine for HER2-positive advanced breast cancer. New Engl J Med. 2006;355(26):2733-43.

9. Hecht JR, Bang YJ, Qin SK, Chung HC, Xu JM, Park JO, et al. Lapatinib in combination with capecitabine plus oxaliplatin in human epidermal growth factor receptor 2-positive advanced or metastatic gastric, esophageal, or gastroesophageal adenocarcinoma: TRIO-013/LOGiC-a randomized phase III trial. J Clin Oncol. 2016;34(5):443-51.

10. Satoh T, Xu RH, Chung HC, Sun GP, Doi T, Xu JM, et al. Lapatinib plus paclitaxel versus paclitaxel alone in the second-line treatment of HER2-amplified advanced gastric cancer in Asian populations: TyTAN-a randomized, phase III study. J Clin Oncol. 2014;32(19):2039-49.
11. Holbro T, Hynes NE. ErbB receptors: directing key signaling networks throughout life. Annu Rev Pharmacol Toxicol. 2004;44:195-217.

12. Wang X, Batty KM, Crowe PJ, Goldstein D, Yang JL. The potential of panHER inhibition in cancer. Front Oncol. 2015;5:2.

13. Zhang K, Sun J, Liu N, Wen D, Chang D, Thomason A, et al. Transformation of NIH 3 T3 cells by HER3 or HER4 receptors requires the presence of HER1 or HER2. J Biol Chem. 1996;271(7):3884-90.

14. Nam HJ, Kim HP, Yoon YK, Hur HS, Song SH, Kim MS, et al. Antitumor activity of HM781-36B, an irreversible Pan-HER inhibitor, alone or in combination with cytotoxic chemotherapeutic agents in gastric cancer. Cancer Lett. 2011;302(2):155-65.

15. Kim TM, Lee KW, Oh DY, Lee JS, Im SA, Kim DW, et al. Phase 1 studies of poziotinib, an irreversible pan-HER tyrosine kinase inhibitor in patients with advanced solid tumors. Cancer Res Treat. 2018;50(3):835-42.

16. O'Donovan N, Byrne AT, O'Connor AE, McGee S, Gallagher WM, Crown J. Synergistic interaction between trastuzumab and EGFR/HER-2 tyrosine kinase inhibitors in HER-2 positive breast cancer cells. Invest New Drugs. 2011;29(5):752-9.

17. Carey LA, Berry DA, Cirrincione CT, Barry WT, Pitcher BN, Harris LN, et al. Molecular heterogeneity and response to neoadjuvant human epidermal growth factor receptor 2 targeting in CALGB 40601, a randomized phase III trial of paclitaxel Plus trastuzumab with or without lapatinib. J Clin Oncol. 2016;34(6):542-9.

18. Eisenhauer EA, Therasse P, Bogaerts J, Schwartz LH, Sargent D, Ford R, et al. New response evaluation criteria in solid tumours: revised RECIST guideline (version 1.1). Eur J Cancer. 2009;45(2):228-47.

19. Simon R. Optimal two-stage designs for phase II clinical trials. Control Clin Trials. 1989;10(1):1-10.

20. Oh DY, Lee KW, Cho JY, Kang WK, Im SA, Kim JW, et al. Phase II trial of dacomitinib in patients with HER2-positive gastric cancer. Gastric Cancer. 2016;19(4):1095-103.

21. Gianni L, Pienkowski T, Im YH, Roman L, Tseng LM, Liu MC, et al. Efficacy and safety of neoadjuvant pertuzumab and trastuzumab in women with locally advanced, inflammatory, or early HER2-positive breast cancer (NeoSphere): a randomised multicentre, open-label, phase 2 trial. Lancet Oncol. 2012;13(1):25-32.

22. Baselga J, Bradbury I, Eidtmann H, Di Cosimo S, de Azambuja E, Aura C, et al. Lapatinib with trastuzumab for HER2-positive early breast cancer (NeoALTTO): a randomised, open-label, multicentre, phase 3 trial. Lancet. 2012;379(9816):633-40.

23. Schneeweiss A, Chia S, Hickish T, Harvey V, Eniu A, Hegg R, et al. Pertuzumab plus trastuzumab in combination with standard neoadjuvant anthracycline-containing and anthracycline-free chemotherapy regimens in patients with HER2-positive early breast cancer: a randomized phase II cardiac safety study (TRYPHAENA). Ann Oncol. 2013;24(9):2278-84.

24. Swain SM, Baselga J, Kim SB, Ro J, Semiglazov V, Campone M, et al. Pertuzumab, trastuzumab, and docetaxel in HER2-positive metastatic breast cancer. New Engl J Med. 2015;372(8):724-34.

25. Tabernero J, Hoff PM, Shen L, Ohtsu A, Shah MA, Cheng K, et al. Pertuzumab plus trastuzumab and chemotherapy for HER2positive metastatic gastric or gastro-oesophageal junction cancer (JACOB): final analysis of a double-blind, randomised, placebocontrolled phase 3 study. Lancet Oncol. 2018;19(10):1372-84.

26. Wainberg ZA, Anghel A, Desai AJ, Ayala R, Luo T, Safran B, et al. Lapatinib, a dual EGFR and HER2 kinase inhibitor, selectively inhibits HER2-amplified human gastric cancer cells and is synergistic with trastuzumab in vitro and in vivo. Clin Cancer Res. 2010;16(5):1509-19.

27. Jackisch C, Welslau M, Schoenegg W, Selbach J, Harich HD, Schröder J, et al. Impact of trastuzumab treatment beyond disease 
progression for advanced/metastatic breast cancer on survivalresults from a prospective, observational study in Germany. Breast. 2014;23(5):603-8.

28. Palle J, Tougeron D, Pozet A, Soularue E, Artru P, Leroy F, et al. Trastuzumab beyond progression in patients with HER2-positive advanced gastric adenocarcinoma: a multicenter AGEO study. Oncotarget. 2017;8(60):101383-93.

29. Makiyama A, Sagara K, Kawada J, Kashiwada T, Hosokawa A, Horie Y, et al. A randomized phase II study of weekly paclitaxel \pm trastuzumab in patients with HER2-positive advanced gastric or gastro-esophageal junction cancer refractory to trastuzumab combined with fluoropyrimidine and platinum: WJOG7112G (T-ACT). J Clin Oncol. 2018;36(15_Suppl):4011.

30. Thuss-Patience PC, Shah MA, Ohtsu A, Van Cutsem E, Ajani JA, Castro $\mathrm{H}$, et al. Trastuzumab emtansine versus taxane use for previously treated HER2-positive locally advanced or metastatic gastric or gastro-oesophageal junction adenocarcinoma (GATSBY): an international randomised, open-label, adaptive, phase 2/3 study. Lancet Oncol. 2017;18(5):640-53.

31. Takegawa N, Nonagase Y, Yonesaka K, Sakai K, Maenishi O, Ogitani Y, et al. DS-8201a, a new HER2-targeting antibody-drug conjugate incorporating a novel DNA topoisomerase I inhibitor, overcomes HER2-positive gastric cancer T-DM1 resistance. Int J Cancer. 2017;141(8):1682-9.

32. Doi T, Shitara K, Naito Y, Shimomura A, Fujiwara Y, Yonemori $\mathrm{K}$, et al. Safety, pharmacokinetics, and antitumour activity of trastuzumab deruxtecan (DS-8201), a HER2-targeting antibodydrug conjugate, in patients with advanced breast and gastric or gastro-oesophageal tumours: a phase 1 dose-escalation study. Lancet Oncol. 2017;18(11):1512-22.

33. Iwasa S, Shitara K, Takahashi S, Park H, Kadowaki S, Modi $\mathrm{S}$, et al. Updated results of phase 1 study of DS-8201a in subjects with HER2-expressing gastric cancer. J Clin Oncol. 2018;36(4_suppl):118.

34. Watson S, Validire P, Cervera P, Zorkani N, Scriva A, Lemay F, et al. Combined HER2 analysis of biopsies and surgical specimens to optimize detection of trastuzumab-eligible patients in eso-gastric adenocarcinoma: a GERCOR study. Ann Oncol. 2013;24(12):3035-9.

35. Pietrantonio F, Caporale M, Morano F, Scartozzi M, Gloghini A, De Vita F, et al. HER2 loss in HER2-positive gastric or gastroesophageal cancer after trastuzumab therapy: Implication for further clinical research. Int J Cancer. 2016;139(12):2859-64.

Publisher's Note Springer Nature remains neutral with regard to jurisdictional claims in published maps and institutional affiliations. 